\title{
Original Article (short paper) \\ Can power and anaerobic capacity reduce according to disordered eating behaviors in cyclists?
}

\author{
Leonardo de Sousa Fortes \\ Lilyan Carla Vaz Mendonça \\ Pedro Pinheiro Paes \\ Universidade Federal de Pernambuco, Recife, PE, Brasil \\ Jeferson Macedo Vianna \\ Universidade Federal de Juiz de Fora, Juiz de Fora, MG, Brasil \\ Fernando Diefenthaeler \\ Universidade Federal de Santa Catarina, Florianópolis, SC, Brasil
}

\begin{abstract}
The present study aimed to compare the power, anaerobic capacity (AC) and performance in a road bicycle race among cyclists with and without risk of disordered eating behaviors (DEB). The sample was selected in a non-probabilistic way, totaling 69 male road cyclists aged between 19 and 30 years. The Wingate test was used to evaluate peak power (PP) and mean power. Time in minutes was adopted to determine performance in a $120-\mathrm{km}$ road cycling race (competitive event). The Eating Attitudes Test (EAT-26) was completed to assess DEBs. The results did not indicate a difference in PP among cyclists with and without risk of DEBs $\left(F_{(2,67)}=3.92 ; p=0.13\right)$. Findings showed a difference in mean power among cyclists with and without risk of DEBs $\left(F_{(2,67)}=36.43 ; p=0.01\right)$. The results revealed a difference in performance in 120-km cycling races among cyclists with and without risk of DEBs $\left(F_{(2,67)}=46.03\right.$; $p=0.01$ ). It could be concluded that DEBs were associated with a lower mean power and performance in a competitive event among male road cyclists, although the same was not true for PP.
\end{abstract}

Keywords: exercise; metabolism; performance; stress.

\section{Introduction}

Cycling is one of the most traditional sports in the world, especially in Europe, where the "Tour de France" is a renowned event worldwide ${ }^{1}$. Cycling is an Olympic sport divided into track (velodrome) and street racing (road), where individuals compete either individually or in teams ${ }^{2}$. Track races include short-distance $(200 \mathrm{~m})$, medium-distance $(\sim 50 \mathrm{~km})$ and longdistance events $(\sim 250 \mathrm{~km})$.

According to Ronnestad and Ellefsen ${ }^{3}$, maximal oxygen uptake $\left(\mathrm{VO}_{2 \max }\right)$, economy of movement and lactic anaerobic power (i.e., in sprints) are the main indicators of performance in road cycling. It is important to note that the difference between victory and defeat in many road cycling events can be determined in the final sprint, which requires great lactic anaerobic power from athletes. Thus, it is important to analyze factors that interfere with the lactic anaerobic power of road cyclists. Road cycling is categorized as an endurance cycle sport ${ }^{4}$.

Ronnestad and Ellefsen ${ }^{3}$ emphasize the importance of identifying factors that interfere with cyclists' performance. According to these authors, certain physical training techniques (i.e., block periodization) and nutritional ergogenic aids tend to have a positive effect. However, certain aspects that have a negative repercussion on the performance of athletes practicing endurance cycle sports have been revealed ${ }^{5}$, such as poor quality of sleep and inadequate recovery. Moreover, recently,
Bratland-Sanda and Sundgot-Borgen ${ }^{6}$ and El Ghoch, Soave, Cafugi and Grave ${ }^{7}$ emphasized the possibility of disordered eating behaviors (DEBs) reducing athletes' performance. Most importantly, to the best of our knowledge, no studies aiming to confirm the statements made by Bratland-Sanda and SundgotBorgen $^{6}$ and El Ghochet al. ${ }^{7}$ have been conducted until now.

DEBs are unhealthy methods used to reduce body mass ${ }^{8}$ : long periods without consuming foods, use of laxatives/diuretics/ appetite suppressants, use of plastic clothes to cause dehydration during physical training sessions, and self-induced vomiting 9 . Evidence reveals that approximately $25 \%$ of male athletes practicing endurance sports adopt DEBs as a way to reduce body mass $^{10-12}$. According to Chapman and Woodeman ${ }^{10}$, trainers frequently associate body mass reduction with improvement in physical performance, thus justifying DEBs among athletes. Furthermore, clothing that accentuates body shape (i.e., cycling clothes) can cause feelings of embarrassment and, as a result, lead athletes to adopting DEBs to reduce body mass 9 .

Durguerianet al. ${ }^{13}$ investigated 11 Olympic lifters. Fast body mass reduction ( $\sim 5 \%$ ) was induced through certain DEBs in the week prior to a competitive event. The present study did not reveal a difference in anaerobic performance after nutritional intervention, thus suggesting that adopting DEBs for fast body mass reduction may not be an effective strategy to maximize athletes' performance. However, until this moment no studies analyzing the relationship between DEBs and 
physical performance have been performed with endurance cycle sport athletes.

From a more practical perspective, this type of investigation contributes to the area of sports science, as the identification of factors that influence athletes' physical performance is considered to be essential. According to what has been described here, the present study compared the power, anaerobic capacity and performance in road cycling races among cyclists with and without risk of DEBs. Consequently, based on the recommendations from Bratland-Sanda and Sundgot-Borgen ${ }^{6}$ and El Ghoch et al. ${ }^{7}$, the following three hypotheses were formulated: a) cyclists at risk of DEBs show lower alactic anaerobic power than those without risk; b) cyclists at risk of DEBs show lower AC than those without risk; and c) cyclists at risk of DEBs show lower performance in road events than those without risk.

\section{Methods}

\section{Participants}

The study sample was selected in a non-probabilistic way, totaling 76 male cyclists aged between 19 and 30 years, participating in the Brazilian national road cycling championship. Cyclists trained three hours per day on average, five times per week. Inclusion criteria were as follows: a) to have been a cycling athlete for at least two years; b) to systematically train road cycling for at least ten hours per week; c) to have signed up for the national championship organized by the Brazilian Cycling Federation; and d) to be available to respond to the questionnaire, and to participate in anthropometric measurements and the anaerobic performance test (Wingate test). However, seven athletes were excluded as they did not fully complete the questionnaire or did not participate in the test. As a result, this study included a final sample of 69 cyclists.

After receiving information about the procedures to which they would be submitted, participants signed an informed consent form, agreeing with the methodological procedures from this study. The procedures adopted in the present study met the Helsinki norms for human research. This research project was approved by the Human Research Ethics Committee.

\section{Experimental procedures}

Authorization was requested from the Brazilian Cycling Federation to perform data collection during a competitive event. Consequently, a meeting was held with cyclists to explain all the ethical procedures of this study.

Data collection was performed in two stages on a single day (Figure 1). In the first stage, athletes responded to the Eating Attitudes Test questionnaire (EAT-26, Bighetti, Santos, Santos, Ribeiro ${ }^{14}$ ) and subsequently participated in the anthropometric measurements (body mass, height and skin folds). It should be emphasized that athletes received the same instructions verbally and their questions were answered. Additionally, written instructions about how to complete the questionnaire were included. In the second stage, athletes performed the AC and power test.

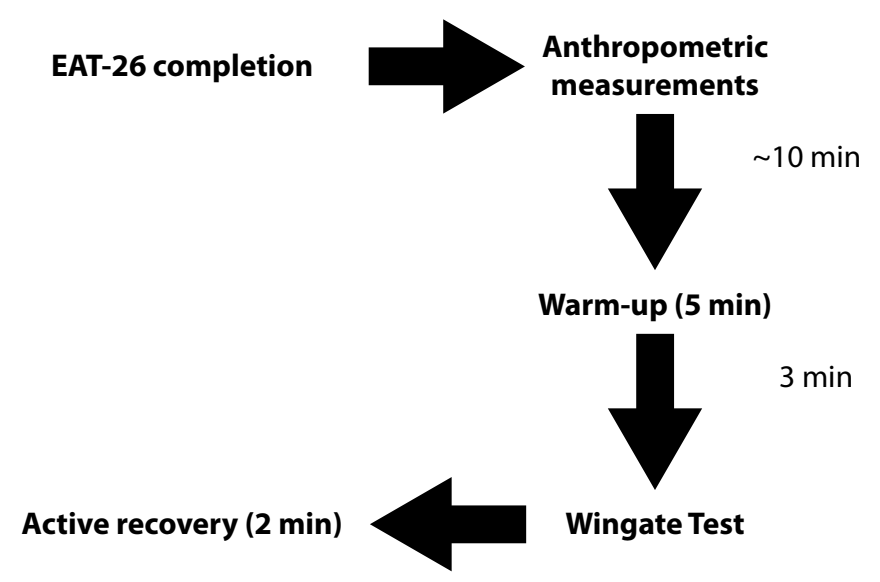

Figure 1. Experimental design of the present study.

\section{Measurements}

The Wingate test was used ${ }^{15}$ to assess peak power(PP) and mean power. According to findings from Bertuzzi, Kiss,Damasceno, Oliveira and Lima-Silva ${ }^{16},{ }^{\mathrm{PP}}$ and mean power can be considered as indicators of anaerobic power and capacity, respectively. A cycle ergometer with an electromagnetic braking system was used (Ergometric 6.0, Cefise, Brazil) to perform the Wingate test. Cyclists remained seated during this test. A warm-up was performed for five minutes, at a cadence of $60 \mathrm{rpm}$ with a constant load of $50 \mathrm{~W}$ and sprints from three to five seconds at the end of every minute, as recommended by Inbar, Bar-Or and Skinner ${ }^{17}$. An interval of three minutes between the end of the warm-up and beginning of the Wingate test was adopted. Consequently, participants were instructed to pedal at maximum speed for 30 seconds against a resistance of $7.5 \%$ of their body mass ${ }^{17}$. Verbal encouragement was given throughout the test. Cyclists were asked to maintain a cadence of pedaling of $120 \mathrm{rpm}$ to begin the Wingate test, aiming to reduce the cycle ergometer's inertia, following the methodology used in the study performed by Moro et al. ${ }^{18}$. External power was calculated every one second. The highest external power in the first five seconds of the Wingate test was used to show PP, while mean power was that obtained during 30 seconds ${ }^{17}$. It should be emphasized that all athletes were familiarized about the Wingate test, thus preventing the learning effect. At the end of this test, cyclists pedaled for two minutes at a cadence of $50 \mathrm{rpm}$ and with a load of $50 \mathrm{~W}$, aiming to recover from fatigue.

Time was calculated in minutes to determine performance in the $120-\mathrm{km}$ road cycling race (competitive event). It should be emphasized that the time of each cyclist for this event was made available by the Brazilian Cycling Federation, after consulting the official race records.

The EAT-26, validated for the Portuguese language by Bighetti, Santos, Santos, Ribeiro ${ }^{14}$, was applied to assess DEBs. This questionnaire is comprised of 26 questions divided into three sub-scales: 1) diet-related to the pathological refusal of high-calorie food and concern with physical appearance; 2) 
bulimia and concern for food - episodes of food compulsion, followed by purgative behavior to lose/control body weight; and 3 ) oral self-control - self-control of food and the environmental and social forces promoting food consumption. For each EAT-26 item, participants had six response options that varied from 0 (few times, almost never and never) to 3 (always). The EAT-26 score is obtained from the sum of its items. The higher the score, the greater the risk of eating disorders. Moreover, it is possible to classify respondents according to the risk of such disorders, i.e., scores equal to or higher than 21 in the EAT-26 indicate risk of triggering eating disorders. In the validation study, Bighetti, Santos, Santos, Ribeiro ${ }^{14}$ indicated an internal consistency of 0.82 . Internal consistency was assessed by Cronbach's alpha and found to be 0.86 for the present sample.

A portable scale (Tanita ${ }^{\circledR}$ BC-601, São Paulo, Brazil) and stadiometer $\left(\right.$ Welmy ${ }^{\circledR}$, Santa Bárbara do Oeste, Brazil) were used to determine body mass and height, respectively. Body mass index (BMI) was calculated according to the following formula: $\mathrm{BMI}=$ body mass $(\mathrm{kg}) /$ height $(\mathrm{m})^{2}$. Body density was determined through the skinfold thickness technique with a scientific caliper (Lange ${ }^{\odot}$, Washington, USA), including tricipital, pectoral and subscapular skinfolds and adopting Jackson and Pollock's protocol (1978) ${ }^{19}$. The International Society for the Advancement of Kinanthropometry's ${ }^{20}$ standards (2013) were used to measure skinfolds. Percentage of body fat $(\% \mathrm{G})$ was determined with Siri's equation $(1956)^{21}$.

\section{Data analysis}

Levene's test was used to test homoscedasticity, while data sphericity was found through Mauchly's test. When the latter assumption was violated, Greenhouse-Geisser correction was adopted. Due to non-parametric violation, central trend measures (mean) and dispersion (standard deviation and standard error) were used to describe variables in this study. Multivariate covariance analysis (MANOVA) was performed to compare PP, mean power and performance in a $120-\mathrm{km}$ race among cyclists at risk $($ EAT-26 $\geq 21)$ and without risk $($ EAT-26<21) for DEBs. It should be emphasized that age was statistically controlled. The Cohen effect size was used, represented by the letter " $d$ " to show differences from a more practical point of view. According to Thalheimer and Cook's ${ }^{22}$ recommendations, the following criteria were used: $d<0.4=$ small effect size, $0.4 \leq d>0.8=$ moderate effect size, and $d \geq 0.8=$ large effect size. The Pearson correlation was used to relatethe PP, mean power, and performance $120-\mathrm{km}$ race with EAT-26 scores. All data were treated with SPSS 21.0, considering a significance level of 5\%.

\section{Results}

Descriptive data [PP, mean power, performance in the $120-\mathrm{km}$ race, EAT-26, BMI, \%G, age, and training regimen (weekly training frequency $\times$ daily training hours)] are shown in Table 1 .

Findings from this study indicate that $30 \%$ of cyclists revealed risks for DEBs according to EAT-26 scores $(\geq 21)$.
Table 1 Descriptive values (mean/standard deviation) of study variables.

\begin{tabular}{ccc}
\hline Variables & Mean & Standard deviation \\
\hline Peak power $(\mathrm{W})$ & 756.71 & 82.43 \\
Mean power $(\mathrm{W})$ & 567.09 & 79.38 \\
Performance in 120-km & 160.75 & 18.09 \\
race (min) & 12.05 & 6.97 \\
EAT-26 & 21.04 & 1.36 \\
BMI (kg/m $\left.{ }^{2}\right)$ & 18.26 & 5.82 \\
$\% \mathrm{G}$ & 22.20 & 1.13 \\
Age (years) & 15.41 & 2.86 \\
\hline Training regimen (hours) &
\end{tabular}

Note. EAT-26 = Eating Attitudes Test; BMI = body mass index; $\% \mathrm{G}=$ percentage of body fat.

MANOVA results (Table 2) did not show a difference in PP between cyclists with and without risk of DEBs $\left(F_{(2,67)}=3.92\right.$; $p=0.13)$, revealing a small effect size $(d=0.1)$. In contrast, findings showed a difference in mean power between cyclists with and without risk of DEBs $\left(F_{(2.67)}=36.43 ; p=0.01 ; d=0.6\right)$. Similarly, the results revealed a difference in performance in the $120-\mathrm{km}$ race between cyclists with and without risk of DEBs $\left(F_{(2,67)}=46.03 ; p=0.01 ; d=0.8\right)$. It should be noted that age showed collinearity with mean power $\left(F_{(2,67)}=7.11 ; p=0.04\right)$, a fact that was not found for $\operatorname{PP}\left(F_{(2,67)}=1.91 ; p=0.29\right)$ or performance in the $120-\mathrm{km}$ race $\left(F_{(2,67)}^{(2,67)}=1.74 ; p=0.26\right)$.

Table 2 Mean and standard error of peak power, mean power and performance in 120-km race according to the dichotomous classifications of the EAT-26.

\begin{tabular}{ccc}
\hline Variable & $\begin{array}{c}\text { Without a risk } \\
(\text { EAT-26 }<2) \\
(\mathbf{n}=\mathbf{4 8})\end{array}$ & $\begin{array}{c}\text { With a risk } \\
(\mathbf{E A T - 2 6 \geq 2 1 )} \\
(\mathbf{n}=\mathbf{2 1})\end{array}$ \\
\hline Peak power (W) & $742(6)$ & $745(6)$ \\
Mean power (W) & $569(6)^{\mathrm{a}}$ & $524(5)$ \\
Performance in 120-km race (min) & $9,532(78)^{\mathrm{a}}$ & $9,706(81)$ \\
\hline
\end{tabular}

Note. EAT-26 = Eating Attitudes Test; ${ }^{\mathrm{a}} \mathrm{p}<0.05$ related to the "with a risk" group.

The findings a revealed statistically significant relationship of mean power $(R=0.38 ; p=0.01)$ and performance $120-\mathrm{km}$ race $(R=0.49 ; p=0.001)$ with DEBs. However, no relationship was found between PP and DEBs $(R=0.08 ; p=0.32)$.

\section{Discussion}

The present study aimed to compare the power, anaerobic capacityand performance in a road cycling event among cyclists with and without DEBs. The results of this study revealed a lower mean power and performance in this event among cyclists at a risk of DEBs when compared to those without a risk, whereas PP was similar between both groups, partially corroborating the initial hypotheses. 
Studies have indicated that approximately $25 \%$ of endurance sports athletes are at risk ofDEBs ${ }^{9-11}$, corroborating the findings from the present study. According to Fortes, Ferreira, Oliveira, Cyrino, Almeida ${ }^{12}$, there are peculiarities in the sports context that can cause one to show DEBs, such as pressure from trainers aiming to achieve better results and clothing that accentuates body shape. It seems that athletes usually associate sports performance optimization with body mass reduction ${ }^{9}$. Thus, some athletes can adopt DEBs as a strategy to quickly reduce body mass. However, it should be emphasized that the adoption of DEBs can be negatively associated with anaerobic performance ${ }^{6}$.

The results of the present study did not reveal a difference in PP between cyclists with and without DEBs, opposing the hypotheses initially formulated. According to El Ghoch,Soave, Cafugi, $\mathrm{Grave}^{7}$, muscle power can be reduced in athletes who show DEBs. El Ghoch, Soave, Cafugi, Grave ${ }^{7}$ emphasize that the adoption of DEBs can lead to a reduction in the speed that motor neurons inherent in high-endurance muscle fibers are recruited and a reduction in the speed of action of the creatine kinase enzyme. However, considering the fact that PP is an indicator of alactic anaerobic metabolism ${ }^{16}$ and that such metabolism lasts approximately five seconds $^{23}$, it is possible to assume that DEBs may not affect alactic anaerobic power in the lower limbs. It should be noted that some scientific findings did not reveal a change in alactic anaerobic power after dehydration or fast body mass reduction ${ }^{13}$, which, in a way, can explain the results for PP in the present study. Most importantly, more studies are required to clarify the relationship between alactic anaerobic power and DEBs.

With regard to mean power, the results indicated a difference between cyclists with and without DEBs. Cyclists at risk of such disorders showed a lower mean power when compared to those without risk of this, corroborating the hypotheses of the present study. It should be emphasized that a moderate effect size was found, which means reasonable probability for this to be true for the population of road cyclists. According to El Ghoch,Soave, Cafugi, Grave ${ }^{7}$, the resynthesis of muscle glycogen can be compromised in athletes who adopt DEBs, aiming to reduce body mass. Thus, considering that anaerobic capacity requires a high level of stored muscle glycogen ${ }^{24}$, it could be assumed that adopting DEBs can lead to a reduction in mean power in the Wingate test, which may explain the findings from the present study. According to Durguerian,Bougard, Drogou, Sauvet, Chennaoul, Filaire ${ }^{13}$, athletes who quickly reduce body mass through unhealthy methods (i.e., DEBs) can increase their psycho-physiological stress and subjective feeling of recovery and, as a result, become less tolerant to fatigue, which may, with some restrictions, be another explanation for the findings of mean power.

Regarding performance in the $120-\mathrm{km}$ cycling race, findings revealed the difference between cyclists with and without risk ofDEBs. Cyclists at risk of DEBs showed poorer performance in the $120-\mathrm{km}$ race when compared to those without risk ofsuch disorders, corroborating the hypotheses of the present study. It should be emphasized that a large effect size was found, which reveals a high probability that this fact could be inferred to the population of male road cyclists. Performance in a road cycling race is multi-factorial (aerobic fitness, lactic aerobic power, economy of movement, and pacing $)^{3}$. Considering aerobic fitness, it is possible to assume that the adoption of DEBs can have negative effects on aerobic energy pathways. According to some authors ${ }^{9}$, the use of laxatives, diuretics and appetite suppressants results in an increase in blood viscosity, which can reduce cardiac output due to cardiovascular overload ${ }^{8}$. On the other hand, regarding pacing, it could be assumed that DEBs result in lack of control of cadence of intensity throughout long-term physical efforts. Considering the fact that success in road cycling depends on the amount of effort made during the race $^{3}$, DEBs can have a negative effect on the pacing strategy adopted by cyclists.

From a practical point of view, PP is a good indicator of an athlete's capacity to sprint for a short time, frequently used at certain moments of a road cycling race, especially the last 50 meters ${ }^{4}$. It seems that the capacity for short-distance sprinting can decide whether someone will win or lose in certain cycling events $^{18}$. According to the results of the present study, the adoption of DEBs appears not to affect the capacity for short-distance sprinting among cyclists. On the other hand, according to Ronnestad, Hansen, Hollan, Ellefsen ${ }^{2}$, mean power is an excellent indicator of anaerobic capacity, which is frequently used in road cycling races. Thus, identifying factors that can negatively interfere in mean power is extremely important to inhibit the reduction in anaerobic capacity in cyclists. Considering the results of the present study, cyclists adopting DEBs can reduce their capacity to perform repeated sprints. In this sense, cyclists at risk ofDEBs require more time to recover between two bursts of high-intensity effort. Moreover, performance in long-duration events depends on aerobic fitness and pacing capacity. Thus, considering the findings from the present study to be inherent in cyclists' performance in $120-\mathrm{km}$ races, it is very likely that cyclists who frequently adopt DEBs reduce their cadence during pedaling due to early fatigue.

Although the present study revealed new and interesting findings, there were some limitations: the use of a questionnaire as an assessment tool, so that the results may not reflect reality due to subjective responses; and the doubly indirect method used to assess $\% \mathrm{G}$. Nonetheless, even with such limitations, this study revealed important findings about the effects that DEBs can have on road cyclists' physical performance.

\section{Practical application statement}

In conclusion, DEBs were associated with lower anaerobic capacity and performance in a competitive event among male road cyclists, although the same is not true for alactic anaerobic power. In other words, the results lead to conclude that lower AC and performance are more prevalent in male road cyclists with DEBs.

\section{References}

1. Diefenthaeler F, Tarragô C, Candotti J, Ribeiro J, Oliveira AR. Comparison of absolute and relative phisiological responses of cyclists and triathletes. Rev Bras Med Esporte. 2007;13(3): 182e185e. doi: 10.1590/S1517-86922007000300015 
2. Ronnestad BR, Hansen J, Hollan I, Ellefsen S. Strength training improves performance and pedaling characteristics in elite cyclists. Scand J Med Sci Sports.2015;25(1): e89-e98. doi: 10.1111/sms. 12257

3. Ronnestad BR, Ellefsen HS. Block periodization of highintensity aerobic intervals provides superior training effects in trained cyclists. Scand J Med Sci Sports.2014;24(1): 34-42. doi: 10.1111/j.1600-0838.2012.01485.x

4. Sangali EB, Campos EZ, Gobbo LA, Andrade VL, Papoti M, Freitas-Júnior IF, Figueira TR, Balikian-Júnior P. Relationship between aerobic and performance indexis in short and medium competitions duration in elite cyclists. Rev. bras. cineantropom. desempenho hum. 2013;15(4): 498-506. doi: 10.5007/1980-0037.2013v15n4p498

5. Hellard P, Avalos M, Hausswirth C, Pyne D, Toussaint J, Mujika I. Identifying optimal overload and taper in elite swimmers over time. J Sports Sci Med. 2013;.12: 668-678.

6. Bratland-Sanda S, Sundgot-Borgen J. Eating disorders in athletes: Overview of prevalence risk factors and recommendations for prevention and treatment. Eur. J. Sport Sci. 2012;1(1): 1-10. doi: 10.1080/17461391.2012.740504

7. El Ghoch M, Soave F, Cafugi S, Grave D. Eating disorders, physical fitness and sport performance: A systematic review. Nutrients. 2013;5(5): 5140-5160. doi: 10.3390/nu5125140

8. BritoC. J, Roas AFM, Brito ISS, Marins JCB, Cordova C, Franchini E. Methods of body-mass reduction by combat sport athletes. Int J Sport NutrExercMetab. 2012;22(1): 89-97.

9. Papathomas A, Petrie T. Editorial: Towards a more sophisticated approach to eating disorders in sport research. PsycholSportExerc. 2014;15: 675-679. doi: 10.1016/j.psychsport.2014.06.006

10. Chapman J, Woodman T. Disordered eating in male athletes: a meta-analysis. J. Sports Sci. 2015. in press. doi: 10.1080/02640414.2015.1040824

11. Fortes LS, Almeida SS, Ferreira MEC. Influence of psychological, anthropometric and sociodemographic factors on the symptoms of eating disorders in young athletes. Paidéia.2014;24(57): 21-27. doi: 10.1590/1982-43272457201404

12. Fortes LS, Ferreira MEC, Oliveira SMF, Cyrino ES, Almeida SS. A socio-sports model of disordered eating among Brazilian male athletes. Appetite. 2015;92(1): 29-35. doi: 10.1016/j. appet.2015.05.005

13. Durguerian A, Bougard C, Drogou C, Sauvet F, Chennaoul M, Filaire E. Weight loss, performance and psychological related states in high-level weighlifters. Int J Sports Med. 2015. in press. doi: 10.1055/s-0035-1555852

14. Bighetti F, Santos CB, Santos JE, Ribeiro RPP. Tradução e avaliação do eating attitudes test em adolescentes do sexo feminino de Ribeirão Preto, São Paulo. J. bras. Psiquiatr. 2004;53(6): 339-46.

15. Bar-Or O. The Wingate anaerobic test. An update on methodology, reliability and validity. Sports Med. 1987;4: 381-394, doi: 10.2165/00007256-198704060-00001
16. Bertuzzi R, Kiss MAPDM, Damasceno M, Oliveira RSF, Lima-Silva AE. Association between anaerobic components of the maximal accumulated oxygen deficit and 30-second Wingate test. Braz. J. Med. Biol. Res. 2015. in press. doi: 10.1590/1414-431X20144043

17. Inbar O, Bar-Or O, Skinner JS. The Wingate anaerobic test. Champaign: J Hum Kinet. 1996.

18. Moro VL, Gheller RG, Berneira JO, Hoefelman CP, Karasiak FC, Moro ARP, Diefenthaeler F. Comparison of body composition and aerobic and anaerobic performance between competitive cyclists and triathletes. Rev. bras. cineantropom. desempenho hum. 2013;15(6): 646-655. doi: 10.5007/1980-0037.2013v15n6p646

19. Jackson AS, Pollock ML. Generalized equations for predicting body density of men. Br J Nutr. 1978;40: 497-504.

20. The Internacional Society for Advancement for Kineanthropometry [homepage on the Internet]. Australia: National Library of Australia [cited 2013 Jul 2013]. Available from: http://www. isakonline.com

21. Siri WE. The gross composition of the body. In: Tobias CA, Lawrence JH, editors. Advances in biological and medical physics. New York: Academic Press;1956: 239-80.

22. Thalheimer W, Cook S. How to calculate effect sizes from published research articles: A simplified methodology. 2002. Retrieved November 25, 2014 from http://work-learning.com/ effect_sizes.htm.

23. Bertuzzi RC, Franchini E, Ugrinowitsch C, Kokubun E, LimaSilva AE, Pires FO. Predicting MAOD using only a supramaximal exhaustive test. Int J Sports Med. 2010;31: 477-481. doi: 10.1055/s-0030-1253375

24. Spriet LL. New insights into the interaction of carbohydrate and fat metabolism during exercise. Sports Med. 2014; 44(1): 87-96. doi: 10.1007/s40279-014-0154-1

\section{Corresponding author}

Leonardo de Sousa Fortes

Clóvis Beviláqua, 163/1003 - Madalena - Recife/PE - 50710330. Brazil Programa de Pós-Graduação em Educação Física, Universidade Federal de Pernambuco, Recife, Brasil.

Email: leodesousafortes@hotmail.com

Manuscript received on June 20, 2016

Manuscript accepted on September 14, 2016

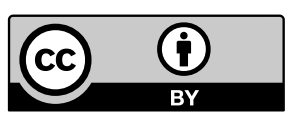

Motriz. The Journal of Physical Education. UNESP. Rio Claro, SP, Brazil - eISSN: 1980-6574 - under a license Creative Commons - Version 3.0 